\title{
„Bedarfsgerechte“ Mittelzuweisung bedarfsgerecht? Ein empirischer Blick auf den Umgang mit schulischen Mitteln und die Sichtweisen von Grundschulleitungen
}

\section{Zusammenfassung}

Die Reduktion des Zusammenhangs von schulischem Erfolg und sozialer Herkunft ist ein fortwährendes bildungspolitisches Ziel - nicht erst seit der Einführung von Vergleichsstudien. Mithilfe des Steuerungsmoments der „bedarfsgerechten“ Mittelvergabe wird seitens der Bildungsverwaltung versucht, diesem Phänomen entgegenzuwirken. Neben dieser Ebene fokussiert der vorliegende Beitrag die Einzelschulebene, indem die Schulleitung als Akteurin im administrativen Umgang mit ihren bedarfsgerechten Mitteln sowie ihre Sichtweise auf diese in den analytischen Mittelpunkt rücken.

Schlüsselwörter: soziale Ungleichheit, bedarfsgerechtes Ressourcenmanagement, Schulleitung, Educational Governance, qualitative Governanceforschung

\section{Are "Needs-based" Allocations Needs-based?}

An Empirical Study of the Handling of School Funds and Views of Primary School Principals

\begin{abstract}
Reduction of the correlation between school success and social background is an ongoing educational policy priority - not only since the introduction of comparative studies. The education administration is attempting to counteract this phenomenon with the help of the steering element of "needs-based" allocation of funds. This article focuses not only on this level, but also on the individual school level, by placing the school principal as an actor in the administrative dealing with these needs-based resources and his or her view of these allocations in the analytical center.
\end{abstract}

Keywords: social inequality, needs-based resource management, school leadership, educational governance, qualitative governance research 


\section{$1 \quad$ Einleitung}

Soziale Ungleichheit und ihre Auswirkungen auf den Bildungserfolg sind ein viel diskutiertes Thema, nicht nur im wissenschaftlichen Diskurs, sondern auch in Bildungspolitik und Öffentlichkeit, was vor allem aus den Ergebnissen der PISAStudie 2000 resultierte. So wurden als Reaktion auf die ersten PISA-Ergebnisse (unverzüglich) öffentlich Maßnahmen vorgestellt, die die „aufgedeckten“ Mängel des deutschen Schulsystems verringern sollen (vgl. KMK, 2002). Eine dieser Maßnahmen ist die bedarfsgerechte Mittelzuweisung, mithilfe derer die jeweiligen Bedingungen und Bedürfnisse der Einzelschule in Form von schulspezifischen zusätzlichen Ressourcen stärker als zuvor Berücksichtigung finden (sollen) (vgl. dazu auch Bos, Pietsch, Gröhlich \& Janke, 2006, S. 149). Auf diese Weise sollen die Schüler*innen sowie die Einzelschulen eine individuelle Unterstützung erhalten, und es soll sich der Egalität von Bildungschancen genähert werden.

Im Umgang mit den schulischen Ressourcen erhält die jeweilige Schulleitung einen eigenen Handlungsspielraum, der individuell ausgestaltet werden kann und muss (vgl. dazu auch Fend, 2008). Der daraus resultierende Möglichkeitsraum für die einzelne Schulleitung eröffnet sich, in Anlehnung an Hummrich, Hebenstreit \& Hinrichsen (2017), aus der subjektiven Wahrnehmung und Ausgestaltung des ,vorgegebenen" Handlungsspielraums. In Bezug auf die bedarfsgerechte Mittelvergabe setzt die Bildungsverwaltung mit ihren Vorgaben in Form von Erlassen und Richtlinien den Handlungsspielraum für die Einzelschule, welcher von dieser individuell verstanden und genutzt wird.

Die Verbindung der Steuerungsmomente auf der Makroebene - bedarfsgerechte Ressourcenzuweisung - und der Mesoebene - schulspezifischer Einsatz dieser Mittel - soll demzufolge soziale Ungleichheit in Bezug auf Bildungschancen verringern.

Anknüpfend an die vorliegenden Beiträge, die die Ressourcenzuweisung und in besonderer Weise die Mittelvergabe mithilfe eines Sozialindex diskutieren, wird das Fehlen einer systematischen Dokumentenanalyse der Ressourcenzuweisungsverfahren sichtbar. Darüber hinaus wurde bisher die Rolle der einzelnen Schulleitung im Umgang mit ihren schulischen Mitteln nicht in den wissenschaftlichen Fokus gerückt (vgl. dazu auch Klemm \& Kneuper, 2019; Nikolai \& Helbig, 2013; Weishaupt, 2016).

Der vorliegende Beitrag setzt an diesen Desideraten an, indem die bedarfsgerechte Ressourcenzuweisung sowie der einzelschulische Umgang damit in den analytischen Fokus gerückt werden. Dazu werden einerseits die bildungspolitischen Richtlinien der Ressourcenzuweisung dahingehend untersucht, welche zusätzlichen Ressourcen durch die Bildungsverwaltung zur Reduktion von sozialer Ungleichheit zur Verfügung ge- 
stellt werden. Andererseits wird den Fragen nachgegangen, wie Grundschulleitungen ${ }^{1}$ die bedarfsgerechte Ressourcenzuweisung einschätzen und welche Strategien sie im administrativen Umgang mit ihren Mitteln aufweisen. Dabei ist anknüpfend an die Makroebene auch zu fragen, inwiefern die jeweiligen bildungspolitischen Vorgaben und Ressourcenzuweisungsverfahren den Umgang der Schulleitungen und somit die Mesoebene leiten, was länderspezifische Umgangsweisen mit den Ressourcen in der Schule impliziert. Dazu wurden Expert*inneninterviews mit Grundschulleitungen an Schulen in sozial deprivierter Lage geführt, da diese andere Bedingungen beispielsweise in Bezug auf Lernmöglichkeiten und Entwicklungschancen aufweisen und bieten (können) (vgl. Morris-Lange, Wendt \& Wohlfarth, 2013, S. 7 f.; dazu auch Klein, 2018) und aufgrund ihrer Schüler*innenschaft in besonderer Weise durch zusätzliche bedarfsgerechte Mittel unterstützt werden (vgl. exemplarisch Beierle, Hoch \& Reißig, 2019, S. 47 ff.).

Mithilfe des angewandten Designs sollen zusammenfassend die steuernde bildungspolitische Ebene und die Einzelschulebene im Kontext ihrer Rekontextualisierung der bildungspolitischen Vorgaben und Zuweisungen miteinander verbunden werden.

Daraus folgend wird im ersten Schritt die bedarfsgerechte Ressourcenzuweisung und somit das Steuerungsmoment auf der Makroebene, unter Verwendung der entsprechenden bildungspolitischen Richtlinien, in den Fokus gerückt (Kap. 2). Anschließend werden die Strategien des innerschulischen Einsatzes der Ressourcen sowie die schulischen Sichtweisen auf diese mithilfe einer Interviewstudie mit Grundschulleitungen dargestellt (Kap. 3). Eine Einschätzung und Verortung der aufgeführten Ergebnisse im Kontext von sozialer Ungleichheit runden den Beitrag ab (Kap. 4).

\section{Mittelzuweisung im Überblick}

Um einen bundesländerübergreifenden Überblick über diejenigen Ressourcen zu erhalten, die zusätzlich, über den Grundbedarf (Unterrichtsabdeckung) hinaus, den Schulen zur Verfügung gestellt werden und somit soziale Ungleichheit in Bezug auf Bildungschancen reduzieren sollen, wurden die länderspezifischen Richtlinien der Ressourcenzuweisung ${ }^{2}$ des Schuljahres 2014/2015 einer Dokumentenanalyse unterzogen (vgl. Gröschner, im Druck). Unter Anwendung der qualitativen Inhaltsanalyse nach Mayring (2010) wurden diejenigen schüler ${ }^{\star}$ innenbezogenen Aspekte aus den Dokumenten herausgearbeitet, die mit zusätzlichen Ressourcen verbunden werden und keiner Diagnostik (von außen) unterliegen. Mit diesem Vorgehen

1 Die Fokussierung auf Grundschulleitungen liegt in deren „leistungsheterogener“ Schüler*innenschaft begründet, da noch keine schulformspezifische Selektion erfolgte, was sich wiederum auch in der Mittelzuweisung für den Primarbereich der einzelnen Bundesländer widerspiegelt (vgl. Gröschner, im Druck).

2 Für Hessen liegen leider keine Dokumente und somit auch keine Ergebnisse vor. 
ist die Annahme verbunden, dass diese Mittel den Schulen aufgrund ihrer Schüler*innenschaft entweder automatisch oder aufgrund von Angaben der Schulen selbst zugewiesen werden.

Im Rahmen der Analyse konnten folgende übergeordnete Kategorien herausgearbeitet werden: natio-ethno-kulturelle Zugehörigkeit, soziale Herkunft sowie Inklusionspauschale. Letztere beinhaltet zumeist die Förderschwerpunkte Lernen, Sprache sowie emotionale und soziale Entwicklung. Sie impliziert eine pauschale Zuweisung für den Bereich „Inklusion“ und basiert somit auf keiner Diagnostik einzelner Schüler*innen. Die Kategorie natio-ethno-kulturelle Zugehörigkeit schließt, in Anlehnung an Mecheril (2002), die drei Dimensionen „Nation“, „Ethnie“ und „Kultur“ ein, da diese kaum voneinander zu trennen sind. Die Kategorie soziale Herkunft umfasst zum einen die (soziale) Herkunft der Schüler*innen und zum anderen die sozialräumliche Lage der Schule. Verbunden sind damit einerseits (empirische) Verfahren, wie ein Sozial- oder Ortsteilindex, welche dazu führen, dass die einzelne Schule aufgrund ihrer Schüler*innenschaft zusätzliche Ressourcen erhält. Zum anderen werden auch Aspekte, die sich auf die räumliche und „soziale“ Verortung der Schule beziehen, mit zusätzlichen Mitteln bedacht. ${ }^{3}$

Im Bundesländervergleich lässt sich zusammenfassend formulieren, dass alle untersuchten Bundesländer zusätzliche Mittel, die über den Grundbedarf hinausreichen, aufgrund der jeweiligen Schüler*innenschaft zur Verfügung stellen (vgl. Tab. 1 auf der folgenden Seite). ${ }^{4}$ Dabei wird davon ausgegangen, dass die zu Kategorien herausgearbeiteten (schüler*innenbezogenen) Aspekte mit einer zusätzlichen Förderbedürftigkeit verbunden sind.

3 Diese drei herausgearbeiteten Kategorien lassen sich auch in den Handlungsfeldern der KMK, die im Anschluss an die Veröffentlichung der ersten PISA-Ergebnisse publiziert wurden, wiederfinden (vgl. KMK, 2002, S. 7).

4 Bei Zuweisungen, die mehrere Kategorien beinhalten, wurden diese mehrfach zugeordnet. 
Tab. 1: Überblick über die Kategorien nach Bundesland

\begin{tabular}{lccc}
\hline Kundesland & $\begin{array}{c}\text { Natio-ethno- } \\
\text { kulturelle } \\
\text { Zugehörigkeit }\end{array}$ & Soziale Herkunft & $\begin{array}{c}\text { Inklusions- } \\
\text { pauschale }\end{array}$ \\
\hline Baden-Württemberg & $\mathrm{X}$ & $\mathrm{X}$ & \\
Bayern & $\mathrm{X}$ & $\mathrm{X}$ \\
Berlin & $\mathrm{X}$ & $\mathrm{X}$ & $\mathrm{X}$ \\
Brandenburg & $\mathrm{X}$ & $\mathrm{X}$ & $(\mathrm{X})^{1}$ \\
Bremen & $\mathrm{X}$ & $\mathrm{X}$ & $\mathrm{X}$ \\
Hamburg & $\mathrm{X}$ & $\mathrm{X}$ & $\mathrm{X}$ \\
Mecklenburg-Vorpommern & $\mathrm{X}$ & $\mathrm{X}$ & $\mathrm{X}$ \\
Niedersachsen & $\mathrm{X}$ & $\mathrm{X}$ & \\
Nordrhein-Westfalen & $\mathrm{X}$ & & \\
Rheinland-Pfalz & $\mathrm{X}$ & & \\
Saarland & $\mathrm{X}$ & $\mathrm{X}$ & $\mathrm{X}$ \\
Sachsen & $\mathrm{X}$ & $\mathrm{X}$ & $\mathrm{X}$ \\
Sachsen-Anhalt & $\mathrm{X}$ & $\mathrm{X}$ & \\
Schleswig-Holstein & $\mathrm{X}$ & & \\
Thüringen & & & \\
\hline Anm & & & \\
\hline
\end{tabular}

Anm.:

1 Seit dem Schuljahr 2016/2017 existiert auch in Bremen eine Inklusionspauschale. Angenommen wird dabei, dass 7,2 Prozent der Schüler*innen einen Förderbedarf im Bereich Lernen, Sprache und Verhalten aufweisen (vgl. Die Senatorin für Kinder und Bildung, 2016, S. 2).

2 Seit dem Schuljahr 2015/2016 wird diese Zuweisung durch einen „Förderpool“ ersetzt, welcher "der Grundschule zur Erfüllung des allgemeinen Förderauftrages und zur Entwicklung eines differenzierten Förderangebotes entsprechend der Heterogenität der Schülerinnen und Schüler zur Verfügung [steht. Dieser ist auch; C. G.] für besondere pädagogische Konzepte aufgrund der regionalen Besonderheiten des Schulstandortes zu nutzen" (Kultusministerium Sachsen-Anhalt, 2017, S. 2).

3 Seit dem Schuljahr 2015/2016 wird eine pauschale Zuweisung von 0,2 LWS für jeweils 15 v. H. Schüler*innen durch den „Förderpool” (siehe Anm. 2) ersetzt (vgl. Kultusministerium Sachsen-Anhalt, 2017, S. 2).

Quelle: eigene Darstellung

Die Vorgaben der Bildungsverwaltung, wie diese Ressourcen unter welchen Bedingungen zugewiesen und innerhalb der Einzelschule eingesetzt werden sollen, sind in den jeweiligen Richtlinien nicht immer ausformuliert. Diese Formulierungen, so die Annahme, haben dabei Auswirkungen auf den Umgang mit diesen auf Einzelschulebene: Eine unkonkrete Ausführung kann einerseits zu einem „breiten“ Handlungsspielraum im Umgang mit der entsprechenden Ressource auf Einzelschulebene führen. Andererseits könnte dies auch zum gegenteiligen Ergebnis führen, da für die Schulleitungen der Prozess der Beantragung und die Anzahl sowie der Einsatz der Ressourcen wenig transparent erscheinen.

Aus diesem Grund wurden die einzelnen Formulierungen der Richtlinien der sieben Bundesländer, die zusätzliche Mittel für alle drei Bereiche zur Verfügung stellen 
(Berlin, Hamburg, Niedersachsen, Nordrhein-Westfalen, Sachsen-Anhalt, SchleswigHolstein und Thüringen), einer weiteren Analyse (strukturierende Inhaltsanalyse) unterzogen. Die Formulierungen wurden dabei jeweils den Ausprägungen „Kategorie wird erwähnt“ (1), „Kategorie wird erwähnt und die Bedingungen der Zuweisung werden dargestellt“ (2) oder „Kategorie wird erwähnt und die Bedingungen der Zuweisung sowie die konkrete Zuweisung an die Schule werden dargestellt“ (3) zugeordnet (vgl. Tab. 2).

Ein vergleichender Blick zwischen den drei induktiv herausgearbeiteten Kategorien zeigt, dass vor allem die Formulierungen und Hinweise bezüglich der Kategorie soziale Herkunft als wenig konkret einzuordnen sind.

Das gegenteilige Ergebnis liegt für die Kategorie Inklusionspauschale vor: Die Zuweisungen für diesen Bereich sind zumeist sowohl in Bezug auf die Bedingungen ihres Erhalts als auch bezüglich der Zuweisungshöhe nachvollziehbar formuliert. Auch die Formulierungen im Kontext der Kategorie natio-ethno-kulturelle Zugehörigkeit implizieren Hinweise zur Beantragung; die konkrete Zuweisung sowie der schulische Einsatz werden jedoch nicht in allen Richtlinien deutlich.

Tab. 2: Ausprägung der Formulierungen nach Kategorie und Bundesland

\begin{tabular}{|c|c|c|c|c|}
\hline Bundes/and & Kategorie & $\begin{array}{l}\text { natio-ethno- } \\
\text { kulturelle } \\
\text { Zugehörigkeit }\end{array}$ & Soziale Herkunft & $\begin{array}{l}\text { Inklusions- } \\
\text { pauschale }\end{array}$ \\
\hline Berlin & & 3 & 3 & 3 \\
\hline Hamburg & & 3 & 3 & 3 \\
\hline Niedersachsen & & 2 & 1 & 3 \\
\hline Nordrhein-Westfalen & & 2 & 2 & 2 \\
\hline Sachsen-Anhalt & & 3 & 1 & 3 \\
\hline Schleswig-Holstein & & 2 & 1 & 3 \\
\hline Thüringen & & 3 & 1 & 3 \\
\hline
\end{tabular}

Anm.: 1 = Kategorie wird erwähnt; 2 = Kategorie wird erwähnt und die Bedingungen der Zuweisung werden dargestellt; 3 = Kategorie wird erwähnt und die Bedingungen der Zuweisung sowie die konkrete Zuweisung an die Schule werden dargestellt.

Quelle: eigene Darstellung 


\section{Mittelzuweisung und der Umgang mit Ressourcen in Grundschulen}

Um die Einschätzungen und Perspektiven auf die sowie den Umgang mit den eigenen schulischen Ressourcen in den Fokus rücken zu können, wurden Expert*inneninterviews mit Grundschulleitungen geführt. Dafür wurden exemplarisch Schulleitungen in den drei Bundesländern Hamburg, Nordrhein-Westfalen und Sachsen-Anhalt ausgewählt, da in diesen Ländern zwar Ressourcen für die drei Bereiche natio-ethno-kulturelle-Zugehörigkeit, soziale Herkunft und Inklusionspauschale zur Verfügung gestellt werden (vgl. Tab. 1), die Zuweisungsgrundlagen aber jeweils different sind (vgl. Tab. 2). Daraus folgend wird durch die Ressourcenzuweisung dem Versuch der Reduktion von sozialer Ungleichheit in Bezug auf Bildungschancen auf sehr unterschiedliche Weise begegnet, was differente Handlungsspielräume für die Einzelschulen vermuten lässt. ${ }^{5}$

Insgesamt liegen der Interviewstudie 16 Expert*inneninterviews aus den zuvor genannten Ländern (fünf aus Hamburg, sechs aus Nordrhein-Westfalen und fünf aus Sachsen-Anhalt) zugrunde. Einbezogen wurden Schulen aus sozial deprivierter Lage, da diese aufgrund ihrer Schüler ${ }^{*}$ innenschaft mehr zusätzliche Ressourcen erhalten (müssten) und dadurch mit diesen umgehen müssen (vgl. Kap. 2). In Hamburg wurden Schulen in die Interviewstudie einbezogen, die einen niedrigen Sozialindex (1 bis 3) aufweisen. In Nordrhein-Westfalen wurde ebenfalls der Sozialindex bei der Auswahl der Schulen angewandt. In Sachsen-Anhalt existiert kein Sozialindex für Schulen; allerdings liegt dort ein Strukturkompass vor (vgl. Institut für Strukturpolitik und Wirtschaftsförderung gGmbH, 2014, S. $5 \mathrm{ff}$.). Dieser bezieht jedoch andere Merkmale als in Hamburg und Nordrhein-Westfalen ein, weshalb zusätzlich der Anteil an Schüleræinnen mit Migrationshintergrund sowie sonderpädagogischem Förderbedarf berücksichtigt wurde - beides Aspekte, die sowohl im Rahmen des Sozialindex in Hamburg (vgl. Bürgerschaft der Freien und Hansestadt Hamburg, 2007) als auch im Rahmen der Ressourcenzuweisungsverfahren der Bundesländer Berücksichtigung finden (vgl. Kap. 2).

5 In Hamburg existiert beispielsweise ein Sozialindex, welcher mithilfe der drei herausgearbeiteten Kategorien die Schüler*innenschaft der Einzelschule in der Zuweisung der Ressourcen jeweils berücksichtigt (vgl. Bürgerschaft der Freien und Hansestadt Hamburg, 2007). Für die Schulen bedeutet dies, dass sie diese Ressourcen ohne zusätzlichen Beantragungsprozess erhalten. In den anderen beiden Bundesländern - Nordrhein-Westfalen und Sachsen-Anhalt - existiert eine solche ,automatische“ Zuweisung nicht. In Nordrhein-Westfalen findet ebenfalls ein Sozialindex bei der Ressourcenzuweisung Berücksichtigung; der Index basiert jedoch, im Gegensatz zu Hamburg, nicht auf Schul-, sondern auf Kreisebene und kommt lediglich bei einzelnen Ressourcen zum Tragen (vgl. Frein, Möller, Petermann \& Wilpricht, 2006, S. 188). In Sachsen-Anhalt werden zwar alle drei herausgearbeiteten Kategorien einbezogen; ein Sozialindex existiert dort jedoch nicht. 
Methodisch orientiert sich das Verständnis von Expert*inneninterviews an Bogner und Menz (2009) und wird als ein theoriegenerierendes Interview verstanden. Die Interviewanalyse erfolgte unter Zuhilfenahme des integrativen Basisverfahrens nach Kruse (2015). Der Anspruch dieses methodischen Verfahrens ist es, „über die Integration verschiedener methodischer Prozessebenen sowie analytischer Perspektiven einen integrativen Grundansatz zu liefern“ (ebd., S. 363; Hervorh. i. O.).

Auf diese Weise konnten die Sichtweisen auf den Ressourcenzuweisungsprozess und die eigenen Mittel sowie der innerschulische Umgang mit den eigenen Ressourcen herausgearbeitet werden. Die nachfolgende Ergebnispräsentation beginnt mit einer allgemeinen Darstellung, wodurch die Spannbreite der Perspektiven und Umgangsweisen insgesamt sichtbar wird. Daran anknüpfend wird der innerschulische Umgang und somit die Aushandlung des eigenen Möglichkeitsraums mithilfe von herausgearbeiteten Schulleitungstypen aufgezeigt.

\subsection{Sichtweisen auf schulische Mittel und der Umgang mit diesen in ihrer Spannbreite}

Die Kenntnis über die und die Auseinandersetzung mit der Ressourcenzuweisung divergiert zwischen den interviewten Schulleitungen. So weisen die Schulleitungen, die sich weniger als (alleinige) Schulleitung, sondern eher als Lehrkraft verstehen, länderübergreifend eine geringere Kenntnis über die Zuweisungsrichtlinien sowie Auseinandersetzung mit diesen auf. Zudem scheinen diese Schulleitungen mit dem Umfang ihrer zugewiesenen Ressourcen eher auszukommen. Weiterhin wird insbesondere bei den Fällen aus Nordrhein-Westfalen deutlich, dass der existierende Sozialindex im Kontext der Mittelzuweisung den interviewten Schulleitungen häufig unbekannt ist. Sie setzen sich zwar (in unterschiedlichem Ausmaß) mit den schulischen Ressourcen auseinander; den Sozialindex als Bezugsgröße kennen sie aber eher nicht.

Der innerschulische Einsatz der zugewiesenen bzw. akquirierten Mittel kann insgesamt als Ausgestaltung des Möglichkeitsraums der einzelnen Schulleitung angesehen werden, weshalb dieser zwischen den interviewten Schulleitungen variiert. Hervorheben lässt sich ein eigener innerschulischer Umgang mit diesen Mitteln auf drei Ebenen: der Unterrichts-, der personellen sowie der administrativen Ebene.

Auf der Ebene des Unterrichts zeigt sich, dass oftmals diejenigen Mittel, die über den Grundbedarf hinausgehen, dazu dienen, den Vertretungsunterricht in den Schulen abzudecken. Dieser Umgang wird vor allem damit begründet, dass dadurch weniger Unterricht für alle Schüler*innen entfällt. Zusätzlich zu dem hieraus resultierenden Wegfall von Unterrichtsausfall wird die Strategie sichtbar, dass auch solches pädagogisches Personal, das offiziell keinen Unterricht 
erteilen darf, wie die Schulsozialarbeiter*innen, zur Unterrichtsvertretung eingesetzt wird. Vor dem Hintergrund unzureichender Ressourcenabdeckung des Grundbedarfs an Lehrkräften findet unter diesen Umständen demzufolge eine Art „Entprofessionalisierung“ statt, indem andere pädagogische Fachkräfte, die nicht über die notwendige formale Qualifikation verfügen, Unterricht vertreten. Darüber hinaus nutzen die interviewten Schulleitungen als Kompensation für unzureichende Lehrer*innenwochenstunden und Ausfälle von Lehrkräften die Veränderung der Klassenstärke bzw. Zusammenlegung von Klassen ebenso wie eine (temporäre) Reduzierung der Stundentafeln. Unter Rückbezug auf die bildungspolitische Idee, mithilfe von zusätzlichen Ressourcen einzelne Schüler*innen(gruppen) zu fördern und so soziale Ungleichheit in Bezug auf Bildungschancen zu verringern, wird auf der Unterrichtsebene folglich die Grenze des Möglichen aufgrund fehlender (Vertretungs-)Mittel erkennbar.

Neben diesem Umgang auf unterrichtlicher Ebene, zeigt sich auch ein individuelles Vorgehen bezüglich des Einsatzes der Ressourcen auf personeller Ebene. Vor allem die Einstellung von zusätzlichem Personal wie Praktikant*innen oder nicht für den Unterricht ausgebildetem (Fach-)Personal wird als weitere Strategie sichtbar. ${ }^{6}$

Auf administrativer Ebene lassen sich ebenfalls Vorgehensweisen finden, die auf die Wahrnehmung und Ausgestaltung des eigenen Möglichkeitsraums und somit auf einen strategischen Umgang mit Budgetierung verweisen: So werden beispielsweise, wo möglich, mehr Bedarfe angegeben, um eine höhere Zuweisung zu erhalten. Dies ist aus der Perspektive der Schulleitungen erforderlich, da die Zuweisung anderenfalls nicht dem wahrgenommenen Bedarf entsprechen würde.

In Bezug auf die Einschätzung der zur Verfügung stehenden Ressourcen wird zwischen der Grundzuweisung, also der Abdeckung des Unterrichts, und zusätzlichen Mitteln, die über die Grundzuweisung hinausgehen, unterschieden. Seitens der Schulleitungen wird in diesem Rahmen vor allem die Auffassung vertreten, dass die Grundzuweisung ausreichend sei. Die zusätzlichen Mittel werden hingegen als zu gering eingeschätzt. Das bedeutet, dass Bereiche wie Sprachförderung, „Inklusion“ oder Doppelbesetzung aus Sicht der Grundschulleitungen nur unzureichend finanziert werden. Zusätzlich zu der als zu gering empfundenen Zuweisung wird diese auch als ungerecht wahrgenommen, dies zum Teil trotz empirischer Berücksichtigung der jeweiligen Schüler*innenschaft (Sozialindex in Hamburg und Nordrhein-Westfalen).

An diese Argumentationslogiken anschließend könnte eine Begründung dafür, dass die Ressourcen für die Grundzuweisung häufig als ausreichend eingeschätzt werden,

6 Auch Helbig und Nikolai (2019) verweisen auf die besonderen Herausforderungen für Schulen mit einem Anteil an Schüler*innen mit Lernmittelbefreiung über 40 Prozent in Berlin im Kontext der Unterrichtsabdeckung und der im Vergleich dazu hohen Einstellung von "Quereinsteigerinnen“ (vgl. dazu auch Richter, Marx \& Zorn, 2018). 
im strategischen Umgang mit diesen liegen. So wird ein Fehlen in diesem Bereich teilweise durch die zusätzlich erhaltenen Mittel (wie Sprachfördermittel) oder anderes pädagogisches Personal ausgeglichen, was den Mangel dieser zusätzlichen Mittel verstärkt und im Kontext von sozialer Ungleichheit zumindest kritisch zu hinterfragen ist. Die Strategien auf allen drei Ebenen verweisen zudem auf eine unzureichende Mittelausstattung insgesamt und somit länderübergreifend auf fehlende Lehrkräfte (vgl. dazu auch Klemm \& Zorn, 2019).

\subsection{Sichtweisen auf schulische Mittel und der Umgang mit diesen im Vergleich}

Um fallübergreifende Orientierungen im Umgang mit den und die Perspektive auf die eigenen Ressourcen aufzeigen zu können, wurden im Kontext der Interviewanalyse Leitungstypen herausgearbeitet. Auf diese Weise werden differente Perspektiven auf die eigenen schulischen Mittel sowie Umgangsweisen mit diesen sichtbar. Die vier länderübergreifenden ${ }^{7}$ Schulleitungstypen sind: die resignierte, die unternehmerische, die gefühlsbetonte sowie die vorübergehende Leitung. Im Analyseprozess zeigte sich, dass vor allem entlang des Selbstverständnisses als Schulleitungen differente Handlungsweisen mit den aufgegriffenen Problematiken bestehen. Das Selbstverständnis als Schulleitung wird dabei weiterhin durch die Sichtweise auf die eigene Schule, die Positionierung(en) innerhalb der Schule, die Wahrnehmung des eigenen Handelns sowie die handlungsleitende Referenz $z^{8}$ bzw. diejenigen Aspekte und/oder Personen, auf die das schulische Handeln ausgerichtet ist, bedingt.

In Bezug auf das eigene Selbstverständnis als Schulleitung nimmt sich die resignierte Leitung stärker als Lehrkraft mit Leitungsfunktionen wahr, wodurch sie aufgrund dieser Doppelfunktion den (eigenen) Erwartungen nicht gerecht werden kann (vgl. dazu auch Wissinger, 2015, S. 60). Davon abgrenzend verstehen sich die unternehmerische Leitung und die gefühlsbetonte Leitung als (alleinige) Leitung und Expertin für die eigene Schule, weshalb auch Eigenverantwortlichkeit im Umgang mit den eigenen schulischen Mitteln für sie kennzeichnend ist. Die vorübergehende Leitung befindet sich eher in einem Konflikt zwischen „Leitung sein müssen“ und „(eigentlich) Lehrkraft sein wollen“.

Im Umgang mit den Ressourcen ist die resignierte Leitung aktiv und fühlt sich für diesen Bereich zuständig. Sie versucht, die Schüler*innen zu fördern, wobei der

7 Die vorübergehende Leitung stellt diesbezüglich jedoch eine Ausnahme dar. Dieser Leitungstyp wurde im Rahmen der vorliegenden Studie nur in Nordrhein-Westfalen sichtbar (vgl. Gröschner, im Druck).

8 Mit der handlungsleitenden Referenz sind auch die Perspektive auf die eigene Schülerinnenschaft sowie die pädagogische Ausgestaltung des Schulalltags verbunden (vgl. dazu auch Gröschner, im Druck). 
Kernunterricht jedoch zumeist im Vordergrund steht. Das Zuweisungsverfahren ist dieser Leitung größtenteils bekannt, und sie nutzt die zugewiesenen Ressourcen individuell für die eigene Schule, was nicht immer den Vorgaben der Bildungsverwaltung entsprechen muss. Sie setzt sich, wenn es aus ihrer Perspektive sinnvoll erscheint, bewusst über die bildungspolitischen Vorgaben hinweg. Länderspezifische Umgangsweisen sind im Kontext der eigenen schulischen Mittel bei diesem Schulleitungstypen nicht hervorzuheben. Die unternehmerische sowie die gefühlsbetonte Leitung vertreten dagegen die Perspektive, dass das gesamte schulische Angebot im Fokus stehen sollte. So setzen sich beide mit den Zuweisungsverfahren auseinander und verwenden diese schulspezifisch, was einen wahrgenommenen Handlungsspielraum impliziert. Bei der unternehmerischen Leitung zeigt sich außerdem, dass das Zuweisungsverfahren für den Umgang mit den Ressourcen bzw. die Rekrutierung zusätzlicher Ressourcen eine (zentrale) Rolle spielt: In Hamburg und Nordrhein-Westfalen schätzen die Schulleitungen die zugewiesenen Ressourcen eher als ausreichend ein und können diese für ihren individuellen Bedarf einsetzen, wobei dieser dabei auch von den Vorgaben der Bildungsverwaltung abweichen kann. Für Sachsen-Anhalt erscheint dies ein wenig anders; hier muss sich vermehrt für die finanzielle Absicherung der Schule eingesetzt werden, damit die Angebote innerhalb des schulischen Alltags weitergeführt werden können. Dies könnte darauf hinweisen, dass Schulen, die ihre Ressourcen (auch) aufgrund eines Sozialindex erhalten, zumindest insofern ausreichend mit Ressourcen seitens der Bildungsverwaltung ausgestattet werden und die zugewiesenen Mittel individuell flexibel einsetzen können, dass sie eine weitere Akquise von Mitteln als nicht notwendig erachten.

Auch bei der gefühlsbetonten Leitung erscheint das Zuweisungsverfahren als ein bedeutsamer Faktor: Wenn sich der Sozialindex nicht in dem Maße wie in Hamburg auf die Zuweisung der Schule auswirkt, wird eine aktive Bemühung um Projekte erkennbar. Allerdings empfindet die gefühlsbetonte Leitung ihre eigene Schule im Vergleich $\mathrm{zu}$ anderen Schulen als insgesamt eher gut ausgestattet.

Für die vorübergehende Leitung sind die Ressourcen vor allem eine Zuweisung von außen, die unabhängig vom Einsatz der Schulleitung geschieht. Ein eigener Handlungsspielraum sowie ein eigener Handlungsbedarf werden von ihr nicht thematisiert, was auf ihre als vorübergehend empfundene Aufgabe verweist. Dieses Thema liegt demzufolge nicht im Fokus ihres Schulleitungshandelns und wird nicht als Aufgabe von Schulleitung wahrgenommen.

Resümierend nehmen die unternehmerische, die resignierte sowie die gefühlsbetonte Leitung durchaus einen (individuellen) Handlungsspielraum in Bezug auf die Ressourcengewinnung und den innerschulischen Umgang mit diesen wahr und nutzen diesen schulspezifisch. Dabei wird zudem den bildungspolitischen Vorgaben bewusst nicht immer entsprochen und der Ressourceneinsatz den (aus der Perspektive der Schulleitungen) individuellen Bedürfnissen der eigenen Schule angepasst. 


\section{Diskussion und Ausblick}

Im Ergebnis zeigt sich die Bedeutung der einzelnen Schulleitung im Umgang mit ihren Ressourcen. Ein individueller, von den Vorgaben abweichender Einsatz der Ressourcen ließ sich vor allem im Kontext der Förderressourcen herausarbeiten. In Rückbindung an die Dokumentenanalyse könnte eine Erklärung für den „flexiblen" schulischen Einsatz der zusätzlichen Ressourcen darin liegen, dass auch die Dokumente diesbezüglich teilweise undeutlich und offen formuliert sind. Unter diesen Umständen entsteht ein (breiter) Interpretationsspielraum sowohl auf der Ebene der Bildungsverwaltung im Kontext der Zuweisung als auch auf Einzelschulebene. Auf letzterer wird dieser Handlungsspielraum je nach Schulleitungstyp unterschiedlich verhandelt und ausgestaltet.

Interessant ist an dieser Stelle, dass die Schulleitungen vor allem die Höhe der Grundversorgung als ausreichend einschätzen, während sie die hier im Mittelpunkt der Akquise und Vergabe stehenden zusätzlichen Mittel als zu gering wahrnehmen. Dies überrascht, weil die Befunde zeigen, dass die zusätzlichen Mittel oftmals für Unterrichtsvertretung zweckentfremdet werden und damit die unzureichende Grundausstattung kompensieren. Die bildungspolitische Maßnahme, zusätzliche Mittel zur Förderung einzelner Schülerinnengruppen zur Verfügung zu stellen, um so die Egalisierung von Bildungschancen zu befördern, verfehlt an dieser Stelle ihre Intention (vgl. dazu auch Helbig \& Nikolai, 2019).

Übergreifend ist anhand der vorliegenden Befunde zudem die grundsätzlichere Frage zu stellen, ob eine „Zweckentfremdung“ wie der Einsatz von Förderressourcen als Vertretungsmittel möglicherweise soziale Ungleichheit in Bezug auf Bildungschancen verstärkt. Denn beispielsweise werden Schüler*innen mit einem Defizit in der deutschen Sprache einerseits als zusätzlich förderbedürftig angesehen, weshalb ihnen zusätzliche Ressourcen zuerkannt werden; andererseits werden ihnen aber die Mittel, die zur Reduktion ihres Defizits führen sollen, nicht zur Verfügung gestellt. Diese strategischen innerschulischen Praktiken sind dabei, auch anknüpfend an Gomolla und Radtke (2009), als institutionell diskriminierende Strukturen zu betrachten. Zudem ist dieser innerschulische Einsatz der (Förder-)Ressourcen auch im Kontext des Lehrkräftemangels im Grundschulbereich (vgl. Klemm \& Zorn, 2019) kritisch zu diskutieren: So ist die Intention, mithilfe zusätzlicher Ressourcen den Zusammenhang zwischen sozialer Ungleichheit und Bildungserfolg $\mathrm{zu}$ verringern, unter diesen Umständen kaum realisierbar.

Im Kontext der Frage, wie soziale Ungleichheit in Bezug auf Bildungschancen reduziert werden kann, ist auch der nachfolgende Aspekt zu berücksichtigen: Im Bundesländervergleich zeigt sich, dass der Erhalt von einzelnen Ressourcen, die über eine Grundversorgung hinausgehen, teilweise durch die Schule selbst bean- 
tragt werden muss, wenn diese nicht zum Beispiel aufgrund eines schulspezifischen Sozialindex (Hamburg) zugewiesen werden. Dieses Ergebnis impliziert, dass sich die Schulleitung in diesem Fall aktiv mit den Möglichkeiten der Beantragung sowie den Bedürfnissen der Schüler*innen auseinandersetzen muss, um sowohl die benötigten Ressourcen zu beantragen als auch diese bedarfsspezifisch einzusetzen. Weiterhin bedeutet dies auch, dass Schulleitungen angehalten sind, mithilfe von "Problematisierungen“ der eigenen Schüler*innenschaft, im Sinne von Defizitzuschreibungen, zusätzliche Mittel zu beantragen. Die „problemfokussierte“ Schüler*innenschaft wird unter diesen Umständen „Mittel zum Zweck“, was im Kontext der Interviewstudie als Strategie auf administrativer Ebene sichtbar wird. Auch an dieser Stelle ergeben sich Rückfragen an Mechanismen institutioneller Diskriminierung, ebenso wie in Bezug auf den Umstand, dass sich Schulleitungen kaum mit der Ressourcenvergabe und -nutzung auseinandersetzen, wenn sie den Umgang hiermit nicht als ihre Aufgabe betrachten, sondern die Verantwortung hierfür und die entsprechenden Steuerungsprozesse außerhalb verorten - wie beim herausgearbeiteten Typ der vorübergehenden Leitung. Dies kann bedeuten, dass in diesem Fall die Schüler*innen nicht diejenigen Mittel erhalten, die ihnen seitens der Bildungsverwaltung zur Verfügung gestellt werden.

Bezüglich der Einschätzung des Ressourcenzuweisungsprozesses sowie der eigenen Mittel kann abschließend festgehalten werden, dass diese insbesondere auf das eigene Selbstverständnis als Schulleitung sowie die damit verbundenen Sichtweisen zurückzuführen ist. Für bildungspolitische Steuerung implizieren die Ergebnisse zudem Grenzen der Steuerbarkeit, da eben nicht kausal von einem (länderspezifischen) Zuweisungssystem auf einen bestimmten Umgang mit und Einsatz von Ressourcen geschlossen werden kann. So sind die herausgearbeiteten Schulleitungstypen länderübergreifend $\mathrm{zu}$ betrachten, wobei sie jedoch aufgrund differenter administrativer Zuweisungsverfahren unterschiedlich (stark) agieren müssen.

\section{Literatur und Internetquellen}

Beierle, S., Hoch, C., \& Reißig, B. (2019). Schulen in benachteiligten sozialen Lagen. Untersuchung zum aktuellen Forschungsstand mit Praxisbeispielen. Zugriff am 19.02.2020. Verfügbar unter: https://www.dji.de/fileadmin/user_upload/DJI_Schulen_ in_benachteiligten_sozialen_Lagen.pdf.

Bogner, A., \& Menz, W. (2009). Das theoriegenerierende Experteninterview. Erkenntnisinteresse, Wissensformen, Interaktion. In A. Bogner, B. Littig \& W. Menz (Hrsg.), Experteninterviews. Theorien, Methoden, Anwendungsfelder (3., grundlegend überarb. Aufl.) (S. 61-98). Wiesbaden: VS.

Bos, W., Pietsch, M., Gröhlich, C., \& Janke, N. (2006). Ein Belastungsindex für Schulen als Grundlage der Ressourcenzuweisung am Beispiel von KESS 4. Versuch einer Klassifizierung von Schultypen. In W. Bos, H.-G. Holtappels, H. Pfeiffer, H.-G. Rolff \& R. Schulz-Zander (Hrsg.), Jahrbuch der Schulentwicklung, Bd. 14: Daten, Beispiele und Perspektiven (S. 149-160). Weinheim \& München: Juventa. 
„Bedarfsgerechte“ Mittelzuweisung bedarfsgerecht? |

Bürgerschaft der Freien und Hansestadt Hamburg. (2007). Drucksache 18/6927. Zugriff am 04.01.2018. Verfügbar unter: http://www.hamburg.de/contentblob/4025272/5586bd35b bcc4e00f46941ad688d9b28/data/pdf-hh-sozialindex-drucksache-18-6927.pdf.

Die Senatorin für Kinder und Bildung. (2016). Vorlage G32/19 für die Sitzung der Deputation für Kinder und Bildung (städtisch) am 18.05.2016. Entwurf einer Richtlinie für die Ressourcenzuweisung für die Unterrichtsversorgung (unterrichtendes Personal) an den öffentlichen Schulen in der Stadtgemeinde Bremen (Kommunale Zuweisungsrichtlinie). Zugriff am 19.02.2020. Verfügbar unter: https://www.bildung.bremen.de/sixcms/media. php/13/G32-19\%20Kommunale\%20Zuweisungsrichtlinie.pdf.

Fend, H. (2008). Schule gestalten. Systemsteuerung, Schulentwicklung und Unterrichtsqualität. Wiesbaden: VS.

Frein, T., Möller, G., Petermann, A., \& Wilpricht, M. (2006). Bedarfsgerechte Stellenzuweisung - das neue Instrument Sozialindex. Zeitschrift SchulVerwaltung NRW, (6), 188-189. Zugriff am 11.01.2018. Verfügbar unter: https://www.schulministerium.nrw. $\mathrm{de} / \mathrm{docs} / \mathrm{bp} /$ Ministerium/Service/Schulstatistik/Kreissozialindex/Berechnungsweisedes-Sozialindexes.pdf.

Gomolla, M., \& Radtke, F.-O. (2009). Institutionelle Diskriminierung. Die Herstellung ethnischer Differenz in der Schule (3. Aufl.). Wiesbaden: VS. https://doi.org/10.1007/978-3531-91577-7

Gröschner, C. (im Druck). Bedarfsorientierte Mittelzuweisung? Eine qualitative Studie zum Umgang mit sozialer Ungleichheit in der Grundschule.

Helbig, M., \& Nikolai, R. (2019). Bekommen die sozial benachteiligsten Schüler*innen die „besten" Schulen? Eine explorative Studie über den Zusammenhang von Schulqualität und sozialer Zusammensetzung von Schulen am Beispiel Berlins. Zugriff am 07.07.2020. Verfügbar unter: https://bibliothek.wzb.eu/pdf/2019/p19-002.pdf.

Hummrich, M., Hebenstreit, A., \& Hinrichsen, M. (2017). Möglichkeitsräume und Teilhabechancen in Bildungsprozessen. In I. Miethe, A. Tervooren \& N. Ricken (Hrsg.), Bildung und Teilhabe. Zwischen Inklusionsforderung und Exklusionsdrohung (S. 279303). Wiesbaden: VS. https://doi.org/10.1007/978-3-658-13771-7_14

Institut für Strukturpolitik und Wirtschaftsförderung gGmbH. (2014). Strukturkompass Sachsen-Anhalt. Ausgewählte Ergebnisse. Ausgabe 2013. Zugriff am 18.01.2018. Verfügbar unter: https://www.statistik.sachsen-anhalt.de/apps/StrukturKompass/dok/ Strukturkompass-Sachsen-Anhalt-2013.pdf.

Klein, E. D. (2018). Erfolgreiches Schulleitungshandeln an Schulen in sozial deprivierter Lage. Eine Zusammenschau zentraler Grundlagen und Befunde aus der nationalen und internationalen Bildungsforschung. Expertise im Auftrag der Wübben Stiftung (SHIP Working Paper Reihe, 2). Zugriff am 07.07.2020. Verfügbar unter: https://duepublico. uni-duisburg-essen.de/servlets/DerivateServlet/Derivate-44667/Klein_Erfolgreiches_ Schulleitungshandeln.pdf.

Klemm, K., \& Kneuper, D. (2019). Zur Orientierung von Schulausgaben an Sozialindizes ein Bundesländervergleich. Zugriff am 07.07.2020. Verfügbar unter: https://www.fes.de/ themenportal-bildung-arbeit-digitalisierung/artikelseite/zur-orientierung-von-schul ausgaben-an-sozialindizes-ein-bundeslaendervergleich.

Klemm, K., \& Zorn, D. (2019). Impulse, die Schule machen. Steigende Schülerzahlen im Primarbereich: Lehrkräftemangel deutlich stärker als von der KMK erwartet. Zugriff am 19.02.2020. Verfügbar unter: https://www.bertelsmann-stiftung.de/fileadmin/files/BSt/ Publikationen/GrauePublikationen/BST-19-024_Policy_Brief_Schu_lerzahlen-Impul se_die_Schule_machen_6__002_.pdf.

KMK (Sekretariat der Ständigen Konferenz der Kultusminister der Länder in der Bundesrepublik Deutschland). (2002). PISA 2000 - Zentrale Handlungsfelder. Zusammenfassende Darstellung der laufenden und geplanten Maßnahmen in den Ländern. Stand: 
07.10.2002. Zugriff am 16.12.2019. Verfügbar unter: https://www.kmk.org/fileadmin/ Dateien/veroeffentlichungen_beschluesse/2002/2002_10_07-Pisa-2000-Zentrale-Hand lungsfelder.pdf.

Kruse, J. (2015). Qualitative Interviewforschung. Ein integrativer Ansatz (2., überarb. u. ergänzte Aufl.). Weinheim: Beltz Juventa.

Kultusministerium Sachsen-Anhalt. (2017). Unterrichtsorganisation an den Grundschulen. RdErl. des MK vom 20.3.2017-23-84003. Zugriff am 07.07.2020. Verfügbar unter: https://mb.sachsen-anhalt.de/fileadmin/Bibliothek/Landesjournal/Bildung_und_ Wissenschaft/Erlasse/Unterrichtsorganisation_an_den_Grundschulen.pdf.

Mayring, P. (2010). Qualitative Inhaltsanalyse. Grundlagen und Techniken (11., aktual. u. überarb. Aufl.). Weinheim: Beltz.

Mecheril, P. (2002). Natio-kulturelle Mitgliedschaft - ein Begriff und die Methode seiner Generierung. Tertium comparationis, 8 (2), 104-115.

Morris-Lange, S., Wendt, H., \& Wohlfarth, C. (2013). Segregation an deutschen Schulen. Ausmaß, Folgen und Handlungsempfehlungen für bessere Bildungschancen. Zugriff am 19.02.2020. Verfügbar unter: https://www.stiftung-mercator.de/media/downloads/3_ Publikationen/SVR_Studie_Bildungssegregation_Juli_2013.pdf.

Nikolai, R., \& Helbig, M. (2013). Schulautonomie als Allheilmittel? Über den Zusammenhang von Schulautonomie und schulischen Kompetenzen der Schüler. Zeitschrift für Erziehungswissenschaft, 16 (2), 381-403. https://doi.org/10.1007/s11618-013-0359-3

Richter, D., Marx, A., \& Zorn, D. (2018). Lehrkräfte im Quereinstieg: sozial ungleich verteilt? Eine Analyse zum Lehrermangel an Berliner Grundschulen. Zugriff am 07.07.2020. Verfügbar unter: https://www.bertelsmann-stiftung.de/fileadmin/files/BSt/Publikatio nen/GrauePublikationen/Studie_Quereinsteiger_in_Berlin.pdf.

Weishaupt, H. (2016). Sozialindex - Ein Instrument zur Gestaltung fairer Vergleiche: Einführung. In B. Groot-Wilken, K. Isaac \& J.-P. Schräper (Hrsg.), Sozialindices für Schulen. Hintergründe, Methoden und Anwendung (S. 13-25). Münster: Waxmann.

Wissinger, J. (2015). Personalentwicklung als Bedingung der Schulentwicklung. Herausforderungen der schulischen Governance. In J. Berkemeyer, N. Berkemeyer \& F. Meetz (Hrsg.), Professionalisierung und Schulleitungshandeln. Wege und Strategien der Personalentwicklung an Schulen (S. 52-69). Weinheim: Beltz Juventa.

Caroline Gröschner, Dr. des., geb. 1987, wissenschaftliche Mitarbeiterin in der Abteilung Allgemeine Pädagogik des Instituts für Erziehungswissenschaft an der TU Braunschweig.

E-Mail: c.groeschner@tu-braunschweig.de

Korrespondenzadresse: Technische Universität Braunschweig, Institut für Erziehungswissenschaft, Bienroder Weg 97, 38106 Braunschweig 